\title{
Detection of Exudative Maculopathy from Retinal Fundus Images
}

\author{
Sreedevi K S \\ Department of CSE \\ Amrita Vishwa Vidhyapeetham \\ Coimbatore, Tamil Nadu - 641 112, India
}

\author{
Hema P Menon \\ Department of CSE \\ Amrita Vishwa Vidhyapeetham \\ Coimbatore, Tamil Nadu - 641 112, India
}

\begin{abstract}
Diabetic retinopathy is a group of eye problems caused due to diabetes. Longer time a person has diabetes; the higher is his or her chances of developing diabetic retinopathy. One of the stages of diabetic retinopathy is Exudative Maculopathy. Spontaneous progression and regression of exudates causes Exudative Maculopathy.This work focuses on extraction of exudates from retinal fundus images using image processing techniques.
\end{abstract}

\section{Keywords}

Exudative Maculopathy,Local Contrast Enhancement, Fuzzy C Means Clustering,Artificial Neural Network

\section{INTRODUCTION}

Diabetic retinopathy refers to a group of eye problems that people with diabetes may face as a complication. Worldwide, it is a leading cause of blindness among population. Retina is an important part of the eye and vascular changes caused due to diabetes, which is a blood related phenomenon, can lead to partial or total blindness, if it is not detected early. The macula is the central area of the retina, where the images are focused, and enabling one to recognize objects and colors and to read and write. In Exudative maculopathy, anomalous vessels appear that secretes out serum or bleed, leading to formation of scars. Exudative maculopathy can be blocked by laser treatment. It is more common in type 2 diabetes (Adult Onset). It will be useful for doctors if an automated method is available for identifying the exudates at early stage or during progression. This can be achieved by using different image processing techniques for detecting the presence of Exudative maculopathy by extracting the exudates from the retinal fundus image.

This paper is organized as follows: section 2 focuses on literature survey for exudates extraction. Section 3 consists of implementation details which comprises of dataset details, system design, preprocessing, extraction of exudates(level 1) and classification using artificial neural networks (level 2). Section 4 consists of results and analysis. Section 5 gives conclusion and summary.

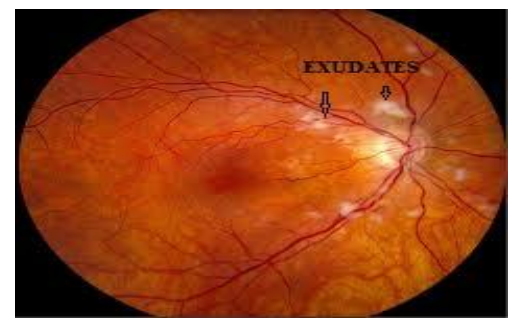

Fig 1: Sample retinal image showing Exudates

\section{LITERATURE SURVEY}

Different methods for extraction of exudates by various authors have been discussed in this section. Akara Sopharak et al. [1] uses an automated method to detect exudates from lowcontrast fundus images of patients with non-dilated pupils using a FCM is proposed. Preprocessing using Contrast enhancement is applied for four features,ie, intensity, standard deviation, hue and als-o a number of edge pixels, are extracted to supply as input parameters to coarse segmentation using FCM. V Vijaya kumari et al.[2] used an automated method involving two steps:optic disk detection and exudates detection. Optic disk is extracted using propagation through radii method. feature extraction, template matching and enhanced MDD classifiers are used for exudates detection and these methods are compared. Alireza Osareh et a. [3] proposed a method for automatic identification of exudates based on Computational Intelligence technique. The colour retinal images were segmented using fuzzy c-means clustering. Feature vector were extracted and classified using multilayer neural network classifier. G S Annie Grace Vimala et al.[4] used retinal images which are pre-processed using Contrast Limited Adaptive Histogram Equalization(CLAHE). The segmentation of preprocessed retinal images is done using K-Means Clustering technique. A dataset of regions is created. A feature set based on colour and texture are extracted for classifying the segmented regions into exudates and non exudates. Classification is done using support Vector Machine. This method was good as it can detect the very small areas of exudates. Sinthanayothin et al. [5] showed detection of Diabetic retinopathy using Recursive Region Growing Segmentation algorithm on $10 * 10$ window moved over the input image Maria Garciaa e al.[6] used Fisher's linear discriminates analysis colour information to perform the classification of retinal exudates. An adaptive threshold algorithm was developed by Leistritz et al. [7] to detect and to measure the exudates in gray value images of patients with diabetic retinopathy. By means of a dynamic thresholding procedure, the usual preprocessing shading correction was omitted since the compensation of irregularities of illumination is implicitly contained in the algorithm. Niemeijer et al. [8] distinguished the bright lesion like exudates, cotton wool spots from colour retinal images. First pixels were classified creating a probability map that contains the probability of each pixel which is a bright lesion. Walter et al [9] identified exudates from green channel of the retinal images according to their gray level variation. The exudates were determined using morphology techniques. T Yamuna et al[10] improved the quality of image using illumuination equalization and contrast limited adaptive equalization(CLAHE). Then candidate extraction is done using shade correction which uses pre processed image and green channel image. Also a top hat transform is applied to extract small details in the image and then image is binarized 
using fixed thresholding.But candidates are not proper representation of these red lesions. So a simple region growing method is applied. Then various stages of abnormalities are classified based on features like standard deviation, entropy, mean etc Adaptive Neuro Fuzzy Inference System(ANFIS).ANFIS classifies the image into normal,mild, severe depending on their severity.Kande et al [11] use two approaches for exudates and optic disk. The centre of optic disk was estimated by finding a point that has maximum local variance. The color morphology in Lab space was used to have homogeneous optic disk region. Optic disk boundary is located using geometric active contour with variational formulation. Preprocessing, Optic disk elimination and Segmentation are done for extracting exudates .The enhanced segments are extracted based on Spatially Weighted Fuzzy cMeans clustering algorithm.

A local contrast enhancement technique invented by Sinthanayothin does not depend on global statistics of the image. It is applied to local areas depending on its mean and variance.This method gave more contrast compared to global contrast enhancement as well as other enhancement techniques. Fuzzy C-Means clustering gave better result than K-Means Clustering.

\section{IMPLEMENTATION DETAILS}

\subsection{Dataset details}

The dataset consists of 25 retinal fundus images. The training dataset consists of both normal images and images with Exudative Maculopathy. Here all images considered are of type JPEG.

\subsection{System Design}

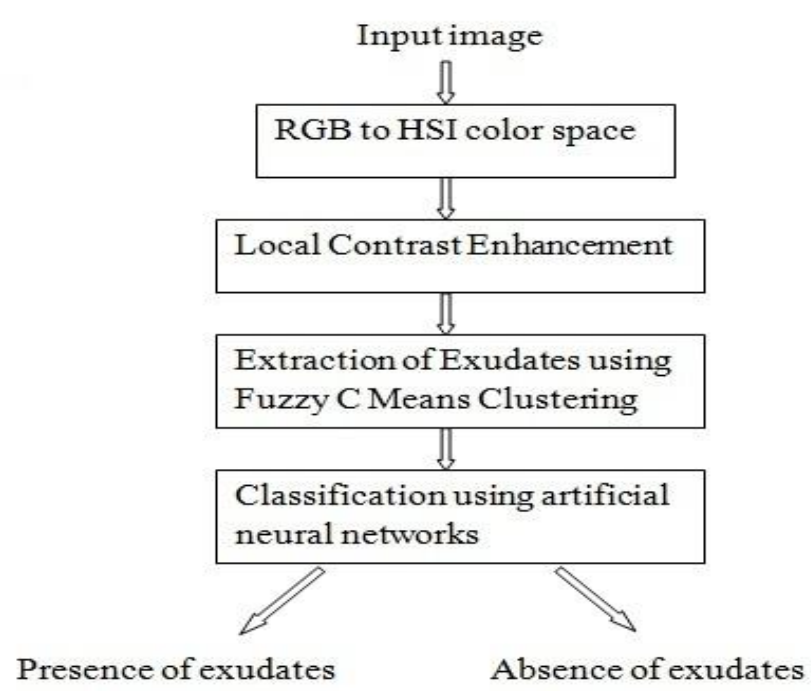

Fig2: General architecture

\subsection{Preprocessing}

Retinal fundus image of the affected eye is taken as the input. In the preprocessing stage the RGB color channel is converted to HSI Color Space. Then a local contrast enhancement method is applied to improve the contrast of the exudates in image. This method is applied on the intensity channel of the fundus image. Here a transformation is applied in small windows of the image such that all intensity values are distributed around the mean and standard deviation.
A Sigmoid function $S_{W}(p)$ is applied in the filtering function:

$$
S_{W}(p)=\left[1+\exp \left(\frac{\mu_{W}-p}{\sigma_{W}}\right)\right]^{-1}
$$

Here $\mu_{W}$ and $\sigma_{W}$ gives the mean and standard deviation within each window. For each pixel $p$ in the original image and a running window $\mathrm{W}$ then the image is filtered using the filtering function to produce a new image I:

$$
I[i, j]=255\left[\frac{S_{W}(p)-S_{W}(\text { Min })}{S_{W}(\operatorname{Max})-S_{W}(\text { Min })}\right]
$$

Min and Max are minimum and maximum intensity values of the whole retinal image.

\subsection{Extraction of exudates}

\subsubsection{Level1-Extraction}

The preprocessed image is then used for segmenting the exudates using Fuzzy C Means clustering. Fuzzy C-Means clustering gives better result for overlapped data set. In KMeans clustering data point must absolutely belong to one cluster center; in FCM data point is assigned membership to each cluster center as a result of which data point may belong to more than one cluster major.

The FCM algorithm used is given below:

1. Initialize $V=\left[v_{i j}\right]$ matrix, $V^{(0)}$

2. at k-step: calculate the centre vectors

$$
\begin{aligned}
& C^{(k)}=\left[c_{j}\right] \text { with } V^{(k)}, \\
& C_{j}=\frac{\sum_{i=1}^{N} v_{i j}^{m} \cdot x_{j}}{\sum_{k=1}^{N} v_{i j}^{m}} \\
& \text { 3. Update } V^{(k)}, V^{(k+1)} v_{i j}=\frac{1}{\sum_{k=1}^{c}\left(\frac{\left\|x_{j}-c_{j}\right\|}{\left\|x_{j}-c_{k}\right\|}\right)^{\frac{2}{m-1}}}
\end{aligned}
$$

4. If $\left\|V^{(k+1)}+V^{(k)}\right\|<\varepsilon$ then stop, otherwise return to step 2.

Here exudates and optic disk are extracted.

\subsubsection{Level 2-Classification of exudates using Artificial Neural networks}

Presence or absence of exudates is checked using artificial neural networks. The main advantage of neural networks is that they are data driven and does not require restrictive assumptions about the form of the basic model. Here a multilayer neural network classifier was used. A feed forward 
architecture was used.The teaching algorithm used is multilayer perceptron. The general steps are:

a. The structure of network is first created where activation functions are chosen and network parameters, biases and weights are initialized.

b. Parameters for training algorithm like error goal, maximum number of epochs are defined.

c. The training algorithm is called.

d. After neural network has been determined, result is first tested by simulating the output of the neural network with the measured input data which is then compared with the measured outputs. Final validation must be carried out with independent data. The number of nodes in the first hidden layer was five and 1 is the number of nodes in the output layer.

Measured input data used are mean, energy, entropy, standard deviation and covariance.

i) Mean: average intensity

$$
m=\sum_{i=0}^{L-1} z_{i} p\left(z_{i}\right)
$$

ii) Energy: is calculated by summing absolute values in a local neighborhood:

$$
L_{e}=\sum_{i=1}^{m} \sum_{j=1}^{n}|c(i, j)|
$$

iii) Entropy: Entropy is a measure of information content. It calculates the randomness of intensity distribution. Entropy is highest when all entries in $P[i, j]$ are of similar magnitude, and small when the entries in $P[i, j]$ are unequal.

iv)Standard Deviation: Shows how spread out intensities are.

v) Covariance: sum of difference between intensity of the central pixel and its neighborhood.

\section{RESULTS AND ANALYSIS}

In this section we compare the results obtained for images containing exudates and non exudates. Fig 3 shows classification result using neural network for abnormal image. The preprocessed Fundus image is converted to HSI Color space. The intensity component alone is extracted. It is then applied to Fuzzy C-Means clustering algorithm. The result consists of three clusters. Since Optic Disc and Exudates are homogenous in color, cluster containing Optic Disk also gets selected for feature extraction. Based on the feature extracted, the neural network is trained for normal and abnormal images. Then image is classified as exudates or non-exudates using artificial neural network. The classifier is needed as along with the exudates the optic disk also gets detected. Hence further segregation of the optic disc and exudates is done using the classifier.

\subsection{Results for Fundus image with Exudates}
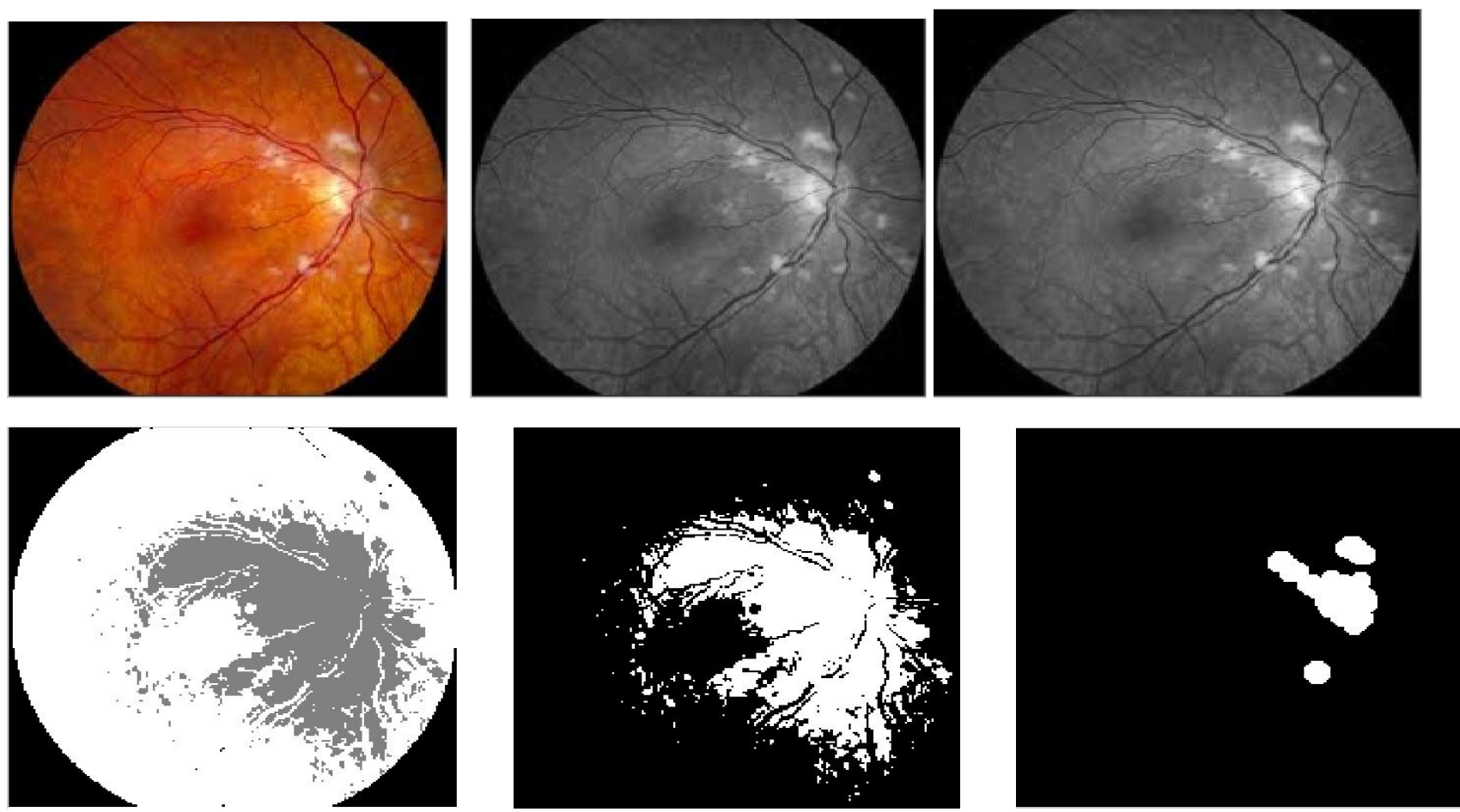

Fig 3: Classification result for an abnormal image containing Exudates:(a)input image (b)intensity image (c)contrast enhanced image (d)fcm 2 clusters (e)exudates (f) segmented exudates and optic disk 


\subsection{Results for Fundus images with}

\section{Exudates}
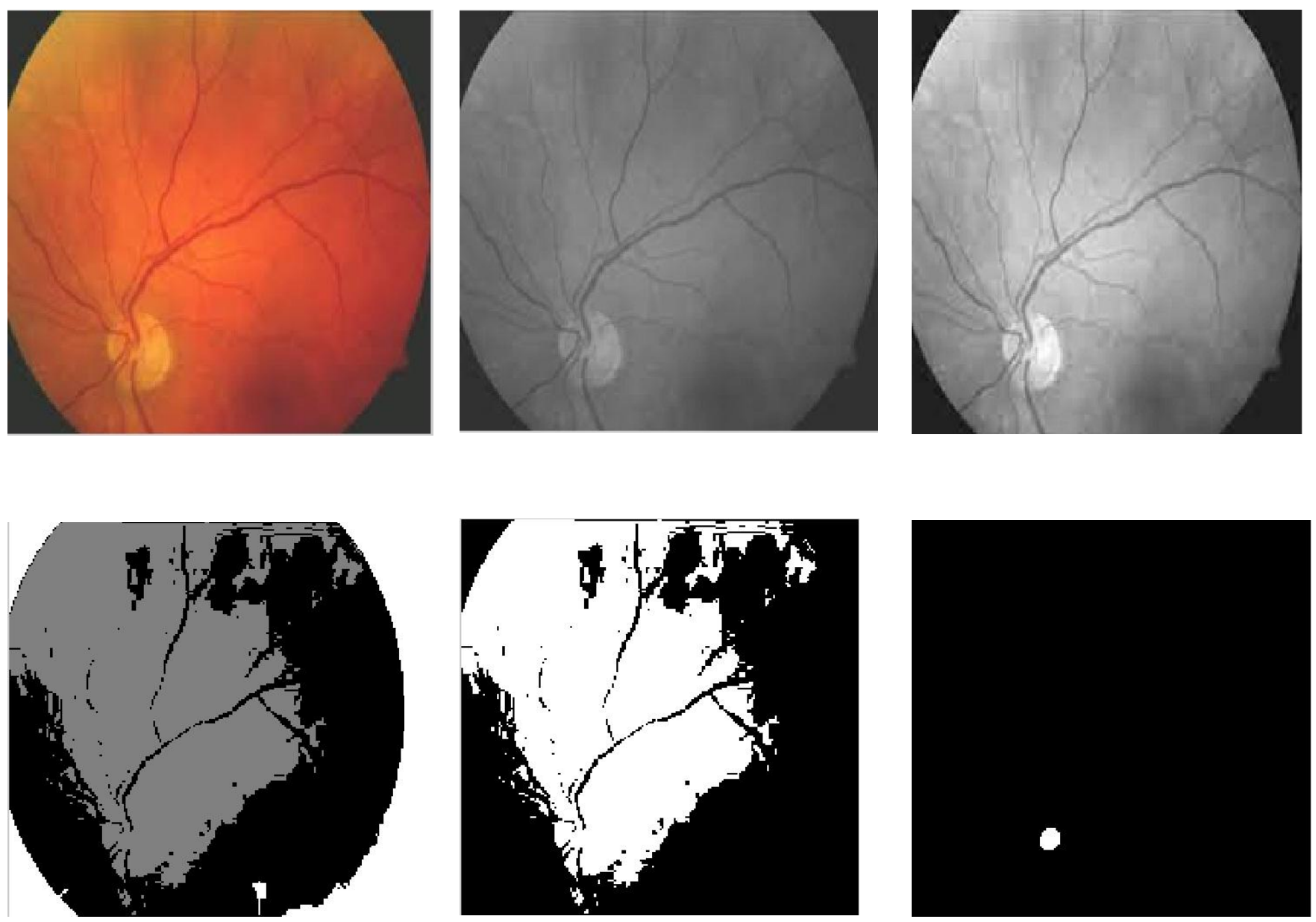

Fig 4: Classification result for normal image containing Exudates :(a)input image (b)intensity image (c)contrast enhanced image (d)fem 2 clusters (e)exudates (f) optic disk

Tables 1 and 2 gives an average values obtained for some of the tested image samples,for the feature that are executed. The extracted features are mean, entropy, standard deviation, covariance, energy for four samples each from normal and abnormal images.

Table 1: Feature extraction for an abnormal image

\begin{tabular}{|l|l|l|l|l|l|}
\hline Sample & Mean & Entropy & $\begin{array}{c}\text { Standard } \\
\text { Deviation }\end{array}$ & Covariance & Energy \\
\hline S1 & 41.4450 & 0.2085 & 23.9197 & 16.3467 & 8289 \\
\hline S2 & 34.9250 & 0.1936 & 21.6794 & 56.6883 & 20758 \\
\hline
\end{tabular}

\begin{tabular}{|l|l|l|l|l|l|}
\hline S3 & 50.2400 & 0.1955 & 23.9300 & 17.1277 & 6985 \\
\hline S4 & 48.9200 & 0.2085 & 31.3742 & 13.5714 & 10048 \\
\hline
\end{tabular}

Table 2: Feature extraction for a normal image

\begin{tabular}{|l|l|l|l|l|l|}
\hline Sample & Mean & Entropy & $\begin{array}{l}\text { Standard } \\
\text { Deviation }\end{array}$ & Covariance & Energy \\
\hline S1 & 5.3550 & 0.0204 & 5.0251 & 0.2309 & 1071 \\
\hline S2 & 9.7450 & 0.0233 & 7.9411 & 0.5723 & 1949 \\
\hline
\end{tabular}




\begin{tabular}{|l|l|l|l|l|l|}
\hline S3 & 16.970 & 0.0627 & 11.7113 & 1.6545 & 3374 \\
\hline S4 & $\begin{array}{l}10.143 \\
3\end{array}$ & 0.0421 & 8.9284 & 0.4566 & 2041 \\
\hline
\end{tabular}

From the above two tables it can be clearly seen that statistical features like mean, entropy, standard deviation, covariance and energy of image containing exudates is much higher than that of the image without exudates. Thus using the statistical features we can then identify the abnormality in the image.

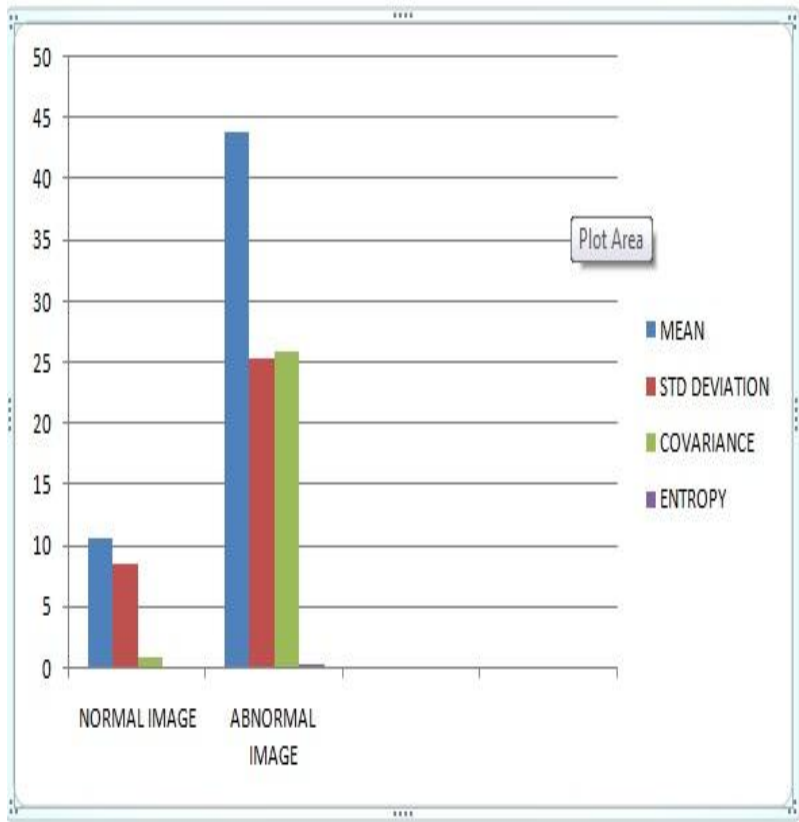

Fig 5: Graph showing the variation in statistical features for images containing exudates and normal image

\section{CONCLUSION AND SUMMARY}

In this paper the exudates that are present in human retinal fundus image was detected successfully using FCM and ANN classifier. The features like mean, standard deviation, entropy ,covariance and energy are used for the classification of exudates and non exudates. The analysis of the features extracted shows that the values obtained will be high for exudates and hence can be used as distinct feature for identification of the presence of exudates. This can be used for the detection of the various stages in the diabetic retinopathy fundus images. The algorithm can be further enhanced by using more features like texture and perceptual measures.

\section{ACKNOLOWLEDGEMENT}

We thank the doctors of Giridhar Eye Institute for their continual support and suggestions throughout the execution of this work.

\section{REFERENCES}

[1] Akara Sopharak , Bunyarit Uyyanonvara, Sarah Barman, Thomas H Williamson. 2008."Automatic detection of diabetic retinopathy exudates from non-dilated retinal images using mathematical morphology methods", Elsevier, Computerized Medical Imaging and Graphics 32 (2008),pp 720-727.

[2] V Vijaya Kumari, N SuriyaNarayanan. 2010. "Diabetic Retinopathy-Early Detection Using Image Processing Techniques"; (IJCSE) International Journal on Computer Science and Engineering Vol. 02, No. 02, 2010, 357-361.

[3] Osareh, A., Mirmehdi, M. Thomas, B. , Markham, R. 2010, " Automatic recognition of exudative maculopathy using fuzzy c- means clustering and neural networks", In Medical Image Understanding Analysis, Claridge, E. ; Bamber ,J. , editors. BMVA Press, pp. 49-52.

[4] G S Annie Grace Vimala,, Dr.Kaja Mohideen. 2012,"An Efficient Approach for Detection of Exudates in Diabetic Retinopathy Images Using Clustering Algorithm" , IOSR Journal of Computer Engineering (IOSRJCE) ,ISSN: 2278-0661 Volume 2, Issue 5, pp 4348.

[5] C. Sinthanayothin, 2000, "Image analysis for automatic diagnosis of Diabetic Retinopathy", World Congress on Information and Communication Technologies, pp. 522532 .

[6] Maria Garciaa, Clara I sancheza, maria I Llopez, daniel Abasoloa, Roberto Horneroa,2008, "A novel automatic image processing algorithm for detection of hard exudates based on retinal image analysis", Medical Engineering and Physics, Volume 30,issue 3,pp350-357.

[7] Leistritz, Lutz; Schweitzer, Dietrich, "Automated Detection and quantification of exudates in retinal images', Proc. SPIE Vol.2298, Applications of Digital Image Processing, pp 690-696.

[8] Niemeijer.M, Abramoff.M.D, Van Ginneken.B, 2007, "Information fusion for Diabetic Retinopathy CAD in Digital color fundus photographs", IEEE Transactions on medical imaging, vo7l. 26, no. 10, pp. 1357-1365.

[9] T. Walter, J. Klein, P. Massin, and A. Erginary, 2002, ”A contribution of image processing to the diagnosis of diabetic retinopathy", detection of exudates in colour fundus images of the human retinall. IEEE Trans. Medical. Imaging,Vol. 21, No. 10, pp.1236-1243.

[10] T Yamuna,S Maheshwari, 2013," Detection of abnormalities in retinal images";IEEE,2013 International conference on emerging trends in computing, communication and nanotechnology;pp.236-240.

[11] Giri Babu Kande, P. Venkata Subbaiah, T. Satya Savithri, 2008, "Segmentation of Exudates and Optic Disk in Retinal Images,"icvgip, 2008 Sixth Indian Conference on Computer Vision, Graphics \& Image Processing, pp.535-542. 